\title{
Welcome to the 10th anniversary issue of Bioanalysis
}

\author{
Sankeetha Nadarajah*,1 \\ ${ }^{1}$ Future Science Group, Unitec House, 2 Albert Place, London N31QB, UK \\ *Author for correspondence: s.nadarajah@future-science.com
}

\begin{abstract}
"Bioanalysts are continuously adapting to advanced technologies, regulatory requirements and the likes, and Bioanalysis endeavors to capture the details and discussions of this continuously evolving field."
\end{abstract}

Accepted for publication: 11 March 2019; Published online: 30 April 2019

Keywords: analytical chemistry • Bioanalysis • publications

It is my absolute pleasure in welcoming you to the 10th anniversary issue of Bioanalysis. Since the launch of the journal in 2009 we have evolved steadily, as has the field of bioanalysis, adapting to the needs of the time from both a bioanalytical and publishing perspective, ensuring we publish quality content in timely and accessible formats for the modern bioanalyst.

\section{The past}

Having worked on the journal for 9 of its 10 years, across the Production, Commissioning and Digital departments, I have personally witnessed the inception and development of ideas through working closely with experts and our dedicated editorial board, the enthusiasm of contributors in the preparation and finalization of quality content, and also the general openness of the field to feedback from peers; understanding the need to push for higher standards at every stage. We are delighted to play a role in converging and presenting global bioanalytical discussions in this manner. Through this anniversary issue, we wanted to provide a $360^{\circ}$ overview of the past, the present and the future of the field through short pieces from a wide variety of authors, covering the breadth of the field. We hope you enjoy the content of this issue as much as we enjoyed compiling it!

\section{The present}

This 10th anniversary issue kicks off with a note from our Senior Editor Neil Spooner, providing a concise coverage regarding the progress of 'bioanalysis' over the last decade - from both a journal and industry perspective [1]. As mentioned in his article we have a dedicated full-time editorial team. To provide a sneak peek into our world, we have notes from our Editorial Director who takes a look at developments of the journal and the wider publishing industry over the past decade [2], while the Senior Editor of our sister website reflects upon the partnership between Bioanalysis and Bioanalysis Zone [3], and how through working collaboratively we have been at the forefront of bringing you bioanalytical advancements and conversations [4].

Bioanalysis works closely with several bioanalytical organizations publishing widely discussed publications including: Conference Reports, Consensus Documents and White Papers. One of our key aims is to compile and converge bioanalytical discussions taking place in an accessible manner, with most such high-impact article types being made free access for all our readers. We are delighted to include contributions from some of our key contributors in this issue.

We have an Industry Update from the AAPS Bioanalytical Community Microsampling subgroup and the European Bioanalysis Forum (EBF), where they provide the output from a survey focused on microsampling for quantitative bioanalysis [5]. A note from the EBF looks at the evolution of our working relationship over the decade and what we can work on to improve and progress [6]. Developments of the Chinese bioanalytical landscape and updates from the China Bioanalysis Forum (CBF) are provided in an editorial [7]. Through a series of interviews with five of its members, the Japan Bioanalysis Forum (JBF) looks back at its history and discusses future plans [8]. 
Asia and Australasia are areas where there has been a spike in bioanalytical developments in recent years and we very much hope to begin to cover more work from these regions with time. We also have a quick overview of 'The 10-year bioanalysis challenge in Mexico' [9]. After a decade of publishing, the White Papers summarizing the conclusions and consensus points from each Workshop on Recent Issues in Bioanalysis (WRIB) meetings, we have the organizers looking beyond WRIB and what to expect from the organization and industry over the next decade [10]. Conference Reports from the annual Global CRO Council (GCC) closed forum are usually among our most top-read content pieces. As such we are pleased to include their latest recommendations for classification of commercial LBA kits for biomarkers in drug development [11] in this anniversary issue.

We consider this unique position of working with many such organizations across the globe our strength and privilege, and very much look forward to further establishing our working relationships, while also making new connections in the future.

To say the field has evolved significantly over the last decade is an understatement, as will be explored in this issue through articles looking at specific areas of bioanalysis. As a direct follow-up to his article published in the very first issue of Bioanalysis, Briscoe reflects on the last 10 years of assessing stability in bioanalysis [12]. MacNeil explores instrumental and technical evolution over the past decade in bioanalysis in his commentary article [13], while van de Merbel explores specifically through the pages of Bioanalysis, to provide a picture of the progress in protein quantification by LC-MS [14].

The diversifying portfolio that drives the exciting evolution of the bioanalysis laboratory is explored from an AstraZeneca perspective by Hawthorne et al. [15]. With the continuously growing interest in the discovery and development of biomarkers to facilitate drug development and improve patient care, Stevenson looks at whether we are ready to evolve our thinking when it comes to biomarker assay validation [16], as Yu and Bashaw provide a regulatory perspective of biomarker bioanalysis during drug development [17]. A broader insight into the regulatory efforts in response to a decade of scientific advancement in bioanalytical methods is provided by Haidar [18]. It is essential that the bioanalytical community keeps updated regarding the multitude of guidance and the associated interpretations around the globe. Lowes and Brown reflect on some of the pivotal points that have impacted regulated bioanalysis over the last decade and what may lie ahead, especially at this crucial time when we have had a handful of guidance/drafts published over the last few months [19].

Bioanalysis has always been keen to explore and drive discussions on the broader aspects of the field, with the aim of putting finer details in context of the bigger picture. For example, looking at bioanalytical work from business, training and outsourcing angles. In this anniversary issue, we are pleased to include a Special Feature in which 11 members of the bioanalytical community provide their thoughts and perspectives on gender parity in bioanalysis - a topic of increasing interest and relevance in STEM today [20-30]. Our respondents explore any obstacles faced as a result of their gender in the field and how these can be overcome, as well as how we could inspire the next generation of scientists to advocate gender parity. We hope this will further drive the discussion at all levels in the field.

\section{The future}

Bioanalysts are continuously adapting to advanced technologies, regulatory requirements and the likes, and Bioanalysis endeavors to capture the details and discussions of this continuously evolving field. We are delighted to have reached this milestone, of course this would not be possible without the support and contributions from all our authors, readers, reviewers and editorial board members, and collaborators - all of whom with we would like to celebrate a decade of Bioanalysis - the journal, and the developments of the field! Here is to the next 10 years!

\section{Acknowledgements}

We would like to thank N Spooner for his unwavering guidance and support of Bioanalysis and Bioanalysis Zone, as well as our previous Senior Editors H Hill and B Booth [31].

Financial \& competing interests disclosure

S Nadarajah is an employee of Future Science Group, Ltd. The author has no other relevant affiliations or financial involvement with any organization or entity with a financial interest in or financial conflict with the subject matter or materials discussed in the manuscript apart from those disclosed.

No writing assistance was utilized in the production of this manuscript. 


\section{References}

1. Spooner N. Bioanalysis: 10 years of progress. Bioanalysis 11(7), 547-549 (2019).

2. Dormer L. Celebrating 10 years of publishing Bioanalysis. Bioanalysis 11(7), 551-553 (2019).

3. Bioanalysis Zone. https://www.bioanalysis-zone.com/

4. Maundrell N. Celebrating the collaboration of Bioanalysis and Bioanalysis Zone. Bioanalysis 11(7), 555-556 (2019).

5. Patel SR, Bryan P, Spooner N, Timmerman P, Wickremsinhe E. Microsampling for quantitative bioanalysis, an industry update: output from an AAPS/EBF survey. Bioanalysis 11(7), 619-628 (2019).

6. Timmerman P. A note from the European Bioanalysis Forum (EBF). Bioanalysis 11(7), 561-562 (2019).

7. Jin F, Tang D, Dong K, Zhong D. New development of the Chinese bioanalytical landscape and update of the China Bioanalysis Forum (CBF). Bioanalysis 11(7), 567-569 (2019).

8. Igarashi H, Koseki N, Arakawa T. The Japan Bioanalysis Forum (JBF): the past 8 years and future perspectives. Bioanalysis 11(7), 611-617 (2019).

9. Marcelín-Jiménez G. The 10-year bioanalysis challenge in Mexico. Bioanalysis 11(7), 575-577 (2019).

10. Garofolo W, Savoie N. Looking beyond the WRIB Decennial Index of the White Papers in Bioanalysis. Bioanalysis 11(7), 563-565 (2019).

11. Islam R, Kar S, Ritzén H. Recommendations for classification of commercial LBA kits for biomarkers in drug development from the GCC for bioanalysis. Bioanalysis 11(7), 645-653 (2019).

12. Briscoe C. Assessing stability in bioanalysis: reflections on the last 10 years. Bioanalysis 11(7), 579-582 (2019).

13. MacNeill R. Instrumental and technical evolution over the past decade in bioanalysis. Bioanalysis 11(7), 601-606 (2019).

14. van der Merbel N. Protein quantification by LC-MS: a decade of progress through the pages of Bioanalysis. Bioanalysis 11(7), 629-644 (2019).

15. Hawthorne G, Henderson N, Hölttä M, Stovold C, Wåhlander Å, Wilson A. Bioanalysis - but not as we knew it: an AstraZeneca perspective of the last 10 years evolution to meet a diversifying portfolio. Bioanalysis 11(7), 595-599 (2019).

16. Stevenson LF. Evolving our thinking on biomarker assay validation - are we ready for the next leap? Bioanalysis 11(7), 571-573 (2019).

17. Yu C, Bashaw ED. Regulatory perspective of biomarker bioanalysis during drug development. Bioanalysis 11(7), 607-610 (2019).

18. Haidar S. Regulatory efforts in response to a decade of scientific advancement in bioanalytical methods. Bioanalysis 11(7), 583-586 (2019).

19. Lowes S, Brown M. Bioanalytical method validation guidance language and a decade of progress. Bioanalysis 11(7), 587-593 (2019).

20. Allen K. Perspectives on gender parity in bioanalysis: an interview with Katryn Allen. Bioanalysis 11(7), 655-656 (2019).

21. Borenstein MR. Perspectives on gender parity in bioanalysis: an interview with Michael R Borenstein. Bioanalysis 11(7), 657-658 (2019).

22. Briscoe C. Perspectives on gender parity in bioanalysis: an interview with Chad Briscoe. Bioanalysis 11(7), 659-660 (2019).

23. Cape S. Perspectives on gender parity in bioanalysis: an interview with Stephanie Cape. Bioanalysis 11(7), 661-662 (2019).

24. DeSilva B. Perspectives on gender parity in bioanalysis: an interview with Binodh DeSilva. Bioanalysis. 11(7), 663-664 (2019).

25. Needham S. Perspectives on gender parity in bioanalysis: an interview with Shane Needham. Bioanalysis 11(7), 665-666 (2019).

26. Patel S. Perspectives on gender parity in bioanalysis: an interview with Shefali Patel. Bioanalysis 11(7), 667-669 (2019).

27. Sims A. Perspectives on gender parity in bioanalysis: an interview with Atreyee Sims. Bioanalysis 11(7), 671-672 (2019).

28. Stevenson L. Perspectives on gender parity in bioanalysis: an interview with Lauren Stevenson. Bioanalysis 11(7), 673-674 (2019).

29. Summerfield S. Perspectives on gender parity in bioanalysis: an interview with Scott Summerfield. Bioanalysis 11(7), 675-676 (2019).

30. Tanna N. Perspectives on gender parity in bioanalysis: an interview with Nikunj Tanna. Bioanalysis 11(7), 677-678 (2019).

31. Hill H, Spooner N. Reflecting on Bioanalysis with the Senior Editors. Bioanalysis 11(7), 557-560 (2019). 
